Ebisu Ebisu

Études japonaises Études japonaises

47 | printemps-été 2012

Catastrophes du 11 mars 2011, désastre de Fukushima : fractures et émergences

\title{
Dany SAVELLI (éd.), Le Japon en Russie : imaginaire, savoir, conflits et voyages, revue Slavica Occitania, $\mathrm{n}^{\circ} 33$
}

\section{Gérard Siary}

\section{OpenEdition}

\section{Journals}

Édition électronique

URL : http://journals.openedition.org/ebisu/541

DOI : 10.4000/ebisu.541

ISSN : 2189-1893

Éditeur

Institut français de recherche sur le Japon à la Maison franco-japonaise (UMIFRE 19 MEAE-CNRS)

Édition imprimée

Date de publication : 1 juin 2012

Pagination : 313-317

ISSN : $1340-3656$

Référence électronique

Gérard Siary, « Dany savelu (éd.), Le Japon en Russie : imaginaire, savoir, conflits et voyages, revue Slavica Occitania, n 33 », Ebisu [En ligne], 47 | printemps-été 2012, mis en ligne le 14 mars 2014, consulté le 22 septembre 2020. URL : http://journals.openedition.org/ebisu/541; DOI : https://doi.org/10.4000/ ebisu. 541

Ce document a été généré automatiquement le 22 septembre 2020.

(c) Institut français de recherche sur le Japon à la Maison franco-japonaise 


\title{
Dany SAVELLI (éd.), Le Japon en Russie : imaginaire, savoir, conflits et voyages, revue Slavica Occitania, $\mathrm{n}^{\circ} 33$
}

\author{
Gérard Siary
}

\section{RÉFÉRENCE}

Dany SAVELLI (éd.), Le Japon en Russie : imaginaire, savoir, conflits et voyages, revue Slavica Occitania, $\mathrm{n}^{\circ}$ 33, 2011, 483 p. <http://w3.slavica-occitania.univ-tlse2.fr>.

1 Voilà une somme sur un sujet neuf encore, toute l'histoire des relations nippo-russes, vue surtout côté russe. D. Savelli («De la matriochka au paradis, en passant par la Sibérie (ou de quelques surprises sur le Japon en Russie)»), le rappelle: «Penser conjointement le Japon et la Russie n'est pas chose si fréquente en Occident» (p.13). Voilà deux pays dits hermétiques. Et pourtant, lorsque dans la seconde moitié du XIX siècle, certains artistes russes composent un style prétendument national à coups d'influences étrangères mais dans le sens de la découverte d'un Orient interne à la Russie, on assiste à la fusion du japonisme et des arts primitifs russes. Et dans le même temps, entre péril jaune et rêve d'Asie nippo-russe, la victoire du Japon sur la Russie fait bouger les lignes : ainsi la défaite oblige les Russes à s'intéresser de plus près à leur adversaire. Des relations culturelles se nouent alors, notamment avec la venue du kabuki en URSS en 1928. La guerre amène les peuples à se connaître un peu plus, en Sibérie, surtout, qui devient zone obligée de rencontre. Ainsi, après la Seconde Guerre mondiale, ce sont dans des camps sibériens où triment les nombreux prisonniers de guerre japonais qui rapporteront chez eux une image de la Russie, loin d'être toujours négative.

2 La première partie (Imaginaire et Savoir) retrace l'histoire de l'idée de Japon en Russie des origines jusqu'en 1945. N. Chtchetkina-Rocher («Le Japon dans l'imaginaire russe des $\mathrm{XVII}^{\mathrm{e}}$ et $\mathrm{XVIII}^{\mathrm{e}}$ siècles: de la terra incognita à la terre promise ») montre qu'avant le 
XIX ${ }^{e}$ siècle, l'image du Japon, tributaire de l'Occident, repose sur un savoir de seconde main, sur des informations déformées, sur « les légendes spécifiques à l'orientalisme onirique » (p. 31). S'y ajoute, à la fin du XviII ${ }^{e}$ siècle, l'utopie des beguny («Fuyants »), ces vieux-croyants qui fuient aux marches de l'empire, font « une lecture mystique de l'ailleurs extrême-oriental» (p.36) et imaginent un Japon qu'ils baptisent Belevodie, nom russe qui se traduit par « Eaux-Blanches ». Le pays est assez ignoré pour que cette utopie prenne son essor.

3 Les études japonaises mettent du temps à gagner un statut institutionnel. Selon V. Alpatov ("La genèse des études japonaises en Russie »), les études japonaises ne dépassent pas le stade d'une discipline pratique dans la seconde moitié du XIX ${ }^{e}$ siècle. Le Japon reste peu connu, voire méprisé. La défaite russe de 1905 change tout. Après 1917, une génération de japonisants surgit, avec de forts projets culturels - initier le public russe à la littérature japonaise -, et des figures majeures comme N. I. Konrad, qui critique le système éducatif japonais. Dans les années 1930, certains de ces savants sont fusillés au chef d'espionnage.

4 L. de Meaux («Port-Arthur et le renouveau des études japonaises en Russie ») insiste sur la visée pragmatique de la première japonologie russe, en lien avec l'État. Après 1905, le directeur de l'Institut de Vladivostok, Dmitri Pozdneïev, oriente les études vers les relations contemporaines. L'essor des études japonaises aide alors à la normalisation des rapports entre les deux États, mais reste confiné au monde savant.

5 La deuxième partie (Littérature et arts) offre le point de vue russe sur l'eurasianisme. V. Molodiakov («L'image du Japon dans la littérature symboliste russe») étudie les auteurs symbolistes russes qui, malgré leur intérêt pour l'art du Japon, mettent en garde contre le péril jaune. Vladimir Soloviev, leur mentor, enjoint la Russie de décider si elle opte pour le Christ ou Xerxès, i.e. l'invasion mongole avec le Japon en tête. Valéri Brioussov reprend de Soloviev l'idée de la lutte séculaire entre atlantisme et eurasianisme. À Andreï Biély revient l'intuition du sens mystique du monde quotidien, l'aigu sentiment de l'invasion mongole, laquelle se manifeste par l'apparition du Nippon dans la ville. Son roman Pétersbourg oppose l'Orient, monde de Xerxès, à la Russie, monde du Christ, sans les séparer vraiment: Apollon Abléoukov, un des protagonistes, est à la fois fervent européen et mongol.

6 R. Uno («À propos des motifs japonisants dans l'œuvre de Pavel Kouznetsov et dans celle des néo-primitivistes russes ») retrace la fortune du japonisme en Russie. Il y arrive non pas via le Japon, mais par le modernisme français alors que ne cesse de grandir l'intérêt pour la culture russe traditionnelle et que se forme le modernisme russe ou néo-primitivisme. Ainsi, le peintre Pavel Kouznetsov évolue vers un primitivisme inspiré des arts primitifs russes ainsi que de l'orientalisme de l'Asie centrale et du japonisme des estampes.

7 S. Soojung Lim (“ "L'esprit du continent” ou le Japon, l'Eurasie et l'Empire dans l'œuvre de Vélimir Khlebnikov ») montre que Khlebnikov aspire à trouver chez les Perses et les Mongols « "un esprit du continent" commun qui puisse les unir à la Russie » et promeut une identité eurasienne dont la Russie, la Chine et le Japon sont les éléments, non sans s'effrayer de voir le Japon devenir une puissance impériale occidentalisée qui entre peu à peu en lutte contre le continent, i.e. la Russie.

8 G. Dudarets («Futabatei Shimei à Pétersbourg») retrace la vie du grand slavisant nippon, Futabatei Shimei 二葉亭四迷: sa formation en russe à l'École supérieure des 
langues étrangères de Tokyo ; son œuvre de traducteur qui initie le public à la Russie ; ses liens avec l'émigration politique russe ; son activité de journaliste pour l'Asahi, qui le met en rapport avec le journaliste Vassili Nemirovitch-Dantchenko, idole de la gauche radicale, et l'amène à Saint-Pétersbourg en 1908-1909.

La troisième partie (D'une scène l'autre) se concentre sur la réception du théâtre japonais par les Russes, en Russie comme au Japon. Y. Kitamura et D. Savelli («L'exotisme justifié ou la venue du kabuki en Union soviétique en $1928 »)$ analysent pourquoi et comment la première tournée du kabuki à l'étranger se fit en cette Union soviétique, pays qui rejetait féodalité et bushidō. Après reconnaissance officielle par le Japon de l'Union soviétique le 20 janvier 1925, cette dernière tente d'améliorer les rapports bilatéraux. L'initiative de la tournée revient à Osanai Kaoru 小山内董, figure de proue du shingeki 新劇, fasciné par Stanislavski et Meyerhold, dont M. Hayashi (« Le théâtre japonais face au théâtre occidental et à la tradition : l'œuvre d'Osanai Kaoru [1881-1928] [Résumé de thèse] ») retrace la carrière. Mais les obstacles à la tournée surgissent au Japon. L'extrême-droite craint que le bolchévisme contamine le théâtre national. L'ambassade soviétique à Tokyo promeut le spectacle et choisit Les Quarante-sept Rōnins, pièce riche en actions, pauvre en dialogues, pour séduire le public. Pour les Soviétiques, plus que la conquête du public russe émerveillé par le kabuki, c'est un pas vers les cercles progressistes nippons. Les périodiques éludent les aspects idéologiques discutables du kabuki, mais Géorgui Astakhov, secrétaire d'ambassade à Tokyo, note que le kabuki, ses samouraïs et son seppuku flattent la vision impérialiste anglo-américaine du Japon : cet exotisme est une marque d'antisoviétisme.

D. Savelli («Un témoignage soviétique de 1936 sur le théâtre japonais: "Le Théâtre japonais" de Boris Pilniak ») édite un petit texte de Boris Pilniak, l'un des rares Russes à avoir vu du kabuki dans les années 1920 et 1930, qui ne revendique aucune compétence savante et livre son expérience spontanée, dans un style adapté à la pression idéologique, où la mention de la scène tournante et des toilettes mixtes est supprimée au profit des persécutions contre le théâtre prolétarien.

11 E. Simonova-Goudzenko («Konstantin Simonov et le théâtre japonais ») évoque les pages de K. Simonov (1915-79), dans son Japon. 46 (1977) sur le kabuki. Ce correspondant de guerre au Japon en décembre 1945 songe que «[1]a passion pour le théâtre kabuki était une des passions de l'art révolutionnaire des années vingt» en raison de l'attirance pour «la force concentrée, la retenue, la concentration de la force », liée selon lui à la passion japonaise du sport.

12 La quatrième partie (L'entre-deux ou la Sibérie) en retrace l'histoire quasi jusqu'à nos jours. V. Datsychen ( La présence japonaise en Sibérie du XVII ${ }^{e}$ siècle aux premières décennies du $\mathrm{xx}^{\mathrm{e}}$ siècle) offre un premier panorama. Les Japonais évitent d'abord la Sibérie pour aller en Russie, mais l'homme d'État Enomoto Takeaki 榎本武揚 (1836-1908), premier ambassadeur à Saint-Pétersbourg, œuvre aux bonnes relations et, après l'ouverture du Transsibérien (1901), les visites officielles suivent. Dès 1893, l'armée japonaise dresse la topographie de la région. L'émigration nippone en Sibérie s'accroît de 603 Japonais en 1890 à 3953 en 1900 sur un total de 5819 Japonais vivant en Russie. La guerre russo-japonaise n'altère ni les bons sentiments réciproques ni la circulation des Japonais. Cela change avec la Première Guerre mondiale et la guerre civile. Le Japon intervient en Sibérie orientale et en Transbaïkalie au service des armées blanches de Koltchak. En 1920, après l'avènement des Soviets, il évacue Irkoutsk, mais pas la Transbaïkalie ni la région de l'Amour. Les Soviets mènent la propagande 
communiste auprès des peuples orientaux pour déloger les forces d'occupation nippones. À partir des années 1920, il n'y a plus guère de Japonais en Sibérie. Tout au plus certains résidents japonais du Mandchoukuo sont-ils autorisés après 1932, à la suite de l'insurrection du général Su Bingwen qui menace la colonie nippone, à se réfugier en Extrême-Orient russe. Dans les années 1930, les autorités soviétiques limitent les passages de Japonais en Sibérie. Le Kremlin ferme le consulat de Novossibirsk en 1937, seul reste encore ouvert le consulat du Mandchoukuo à Tchita où vivent des Japonais à la fin des années 1930.

13 S. Kouznetsov («Les anciens prisonniers de guerre japonais évoquent leur captivité en Sibérie ») tient les captifs de Sibérie pour cas à part. Ils se forment en communauté solidaire, vivent dans l'observance des traditions, révèrent l'Empereur au nom duquel ils se sont rendus, se méfient des autorités soviétiques mais sont curieux du monde russe, et sereins dans l'acceptation de leur destin. Les «bons sentiments et souvenirs que les anciens prisonniers de guerre japonais ont gardé de la Russie et des Russes se sont formés non pas à cause mais en dépit des efforts de l'administration du camp comme de la politique soviétique en général ».

T. Takemoto (« Mémoire et violence dans les Chroniques de l'oiseau à ressort de Murakami Haruki ou de Khalkhin Gol aux camps de Sibérie ») analyse un épisode secondaire du roman de Murakami, qui évoque Khalkin Gol (jap. Nomonhan ノモンハン) avant la bataille de 1939, puis un camp sibérien après la défaite de 1945. Il raille les petits soldats nippons pris dans une guerre étrangère et impuissants. Le lieutenant Mamiya voit un Mongol écorcher vif un soldat japonais sur l'ordre d'un officier soviétique. Le corps du supplicié symbolise la défaite de l'armée du Guandong au profit du nouveau maitre. D'où la désacralisation du mythe du dieu de la guerre cher à l'idéologie nationaliste. Murakami renvoie dos à dos les deux parties : l'URSS communiste ne vaut pas mieux que le Japon nationaliste. Le peuple, décidément, reste passif face au pouvoir qui lui fait tout croire - de la grandeur de l'Asie sous la direction du Japon à la sécurité nucléaire à Fukushima...

Dommage que la période contemporaine, après les années 1930, soit fort peu analysée. On aurait aimé en savoir plus sur la japonologie stalinienne et post-stalinienne, plus aussi sur certains créateurs contemporains qu'inspire le Japon, tel B(oris) Akounine, traducteur de littérature japonaise, amateur de Mishima, mais aussi auteur de polars dont certains se passent au Japon. Un travail d'ensemble sur ces questions fait encore défaut, mais le présent recueil offre un fort beau début dans ce sens.

\section{AUTEURS}

GÉRARD SIARY

Université Montpellier 3, CRCAO UMR 8155 